\title{
A CONDUTA EXPECTANTE NO MANEJO DE MULHERES JOVENS COM NIC 2 É SEGURA?
}

\author{
Mariana K. Bonás*, Diama B. Vale
}

\section{Resumo}

O tratamento excisional de lesões precursoras do colo uterino é uma ferramenta médica importante para impedir sua progressão. Em mulheres jovens com NIC 2 são observadas elevadas taxas de regressão, além disso os tratamentos excisionais aumentam a possibilidade de complicações obstétricas. Este estudo é uma avaliação retrospectiva de mulheres com NIC 2 e menos de 30 anos atendidas no CAISM entre 2012-2017 e que foram manejadas conservadoramente. As taxas de regressão encontradas foram elevadas, especialmente nas mulheres menores de 24 anos. A conduta expectante deve ser adotada em mulheres jovens com NIC 2.

\section{Palavras-chave:}

Neoplasias do Colo do Útero, Neoplasia Intraepitelial Cervical, Papilomavírus Humano.

\section{Introdução}

O câncer do colo do útero é o terceiro tumor mais prevalente na população feminina brasileira. (1) A causa mais comum associada a ele é a infecção persistente pelo vírus HPV. (2) Fatores como tabagismo, idade precoce de início da atividade sexual e outros podem contribuir com o aparecimento e evolução das lesões. (3) O tratamento cirúrgico das lesões precursoras do colo do útero - neoplasias intraepiteliais graus $2 / 3$ (NIC 2/3), aumenta 0 risco de complicações obstétricas nas gestações futuras. (4) O manejo conservador de mulheres jovens com NIC 2 pode ser indicado. Existem dúvidas sobre a segurança dessa conduta, já que estudos mostram taxas de regressão muito variáveis. ${ }^{(5)}$

\section{Resultados e Discussão}

Estudo observacional longitudinal com avaliação retrospectiva de prontuários de todas as mulheres com diagnóstico de NIC2 e menores de 30 anos $(\leq 30)$ atendidas no CAISM/Unicamp entre 2012-2017, em que foi adotada a conduta expectante (seguimento com citologia e/ou biópsia incisional). Foram excluídas gestantes, mulheres com imunodepressão e as com antecedente de lesão precursora. Foram avaliadas as taxas de regressão, persistência e progressão, e a curva de probabilidade de regressão (CPR). As variáveis clínicas foram: idade, idade do início da atividade sexual (IAS), paridade, tabagismo e uso de contracepção hormonal $(\mathrm{CH})$. A CPR foi construída pelo método de Kaplan-Meier, e sua relação com as variáveis analisada pela regressão de Cox, admitindo nível de significância de $5 \%$.

\section{Análise descritiva}

- Foram 65 mulheres incluídas no estudo.

- A idade variou de 14 a 30 anos, média 22,4 anos, desvio-padrão (DP):4,2.

- A média da IAS foi de 15,7 anos, DP:2,0.

- O tempo médio de acompanhamento de 18,0 meses, DP:9,9.

- Eram nuligestas $66,2 \%$, tabagistas $14,1 \%$ e usavam $\mathrm{CH} 61,5 \%$.

\section{Análise das variáveis}

- Nos primeiros 12 meses de acompanhamento, regressão, persistência e progressão do NIC2 foram observadas em respectivamente 28 $(62,2 \%), 12(26,7 \%)$ e $5(11,1 \%)$ das mulheres com até 24 anos ( $\leq 24)$ e em $9(45,0 \%), 8(40 \%)$ e $3(15 \%)$ das mulheres de 25 a 30 anos.

- Nas mulheres $\leq 30$ anos aos 6, 12, 18, 24 e 36 meses as probabilidades de regressão foram respectivamente de $42,0 \%, 63,6 \%, 76,1 \%$ e $84,1 \%$.

- Nenhuma variável clínica foi associada com a CPR $(P>0.05)$.

\section{Conclusões}

Mulheres $\leq 30$ anos apresentaram taxas de regressão de NIC 2 elevadas, especialmente as $\leq 24$ anos. A probabilidade em 24 meses de regressão das mulheres $\leq 30$ anos foi de $76,1 \%$. A conduta expectante deve ser adotada em mulheres jovens com NIC2, desde que 0 seguimento seja rigoroso.

\section{Referências Bibliográficas}

1 INSTITUTO NACIONAL DE CÂNCER. Tipos de Câncer: Colo do Útero. Disponível em: <http://www2.inca.gov.br/ wps/wcm/connect/tiposdecancer/site/home/colo_utero/de finicao>. Acesso em: 04 jul. 2019.

SCHIFFMAN, M. et al. Carcinogenic human papillomavirus infection. Nat Rev Dis Primers, v. 2, p. 16086, 2016.

${ }^{3}$ LEE, C. H. et al. Risk evaluation for the development of cervi

cal intraepithelial neoplasia: development and validation of risk-scoring schemes. Int $\mathrm{J}$ Cancer, v. 136, n. 2, p. 340-9, 2015.

4 INSTITUTO NACIONAL DE CÂNCER. Diretrizes brasileiras para o rastreamento do câncer do colo do útero. 2 ed. rev, p. 83, 2016.

5 LEE, M. H. et al. Outcomes of Conservative Management of High Grade Squamous Intraepithelial Lesions in Young Women. J Low Genit Tract Dis, v. 22, n. 3, p. 212-218, 2018. 